\title{
PAPER
}

\section{Organisation of the sympathetic skin response in spinal cord injury}

\author{
P Cariga, M Catley, C J Mathias, G Savic, H L Frankel, P H Ellaway
}

See end of article for

J Neurol Neurosurg Psychiatry 2002;72:356-360

authors' affiliations

.....................

Correspondence to: Dr P Cariga, Department of Sensorimotor Systems,

Division of Neuroscience and Psychological

Medicine, Imperial College

School of Medicine at

Charing Cross Hospital,

Fulham Palace Road,

London W6 8RF, UK;

Pietro_Cariga@hotmail.com

Received 17 July 2001

In revised form

12 October 2001

Accepted

31 October 2001
Objectives: The sympathetic skin response (SSR) is a technique to assess the sympathetic cholinergic pathways, and it can be used to study the central sympathetic pathways in spinal cord injury (SCI). This study investigated the capacity of the isolated spinal cord to generate an SSR, and determined the relation between SSR, levels of spinal cord lesion, and supraspinal connections.

Methods: Palmar and plantar SSR to peripheral nerve electrical stimulation (median or supraorbital nerve above the lesion, and peroneal nerve below the lesion) were recorded in 29 patients with $\mathrm{SCl}$ at various neurological levels and in 10 healthy control subjects.

Results: In complete SCl at any neurological level, SSR was absent below the lesion. Palmar SSR to median nerve stimuli was absent in complete SCI with level of lesion above T6. Plantar SSR was absent in all patients with complete $\mathrm{SCl}$ at the cervical and thoracic level. In incomplete $\mathrm{SCl}$, the occurrence of SSR was dependent on the preservation of supraspinal connections. For all stimulated nerves, there was no difference between recording from ipsilateral and contralateral limbs.

Conclusions: No evidence was found to support the hypothesis that the spinal cord isolated from the brain stem could generate an SSR. The results indicate that supraspinal connections are necessary for the SSR, together with integrity of central sympathetic pathways of the upper thoracic segments for palmar SSR, and possibly all thoracic segments for plantar SSR.
$\mathrm{T}$ he sympathetic skin response (SSR) is a technique that records changes in skin conductance ${ }^{12}$ after activation of sweat glands in skin areas rich in eccrine glands (commonly palmar and plantar sites) under the neural control of sympathetic cholinergic (sudomotor) fibres. Potentials generated by the SSR can be recorded in response to various stimuli; these include electrical peripheral nerve stimulation, acoustic stimuli, or magnetic stimulation of nerves or the brain, ${ }^{3-5}$ although magnetic stimulation lacks specificity in terms of sensory pathways involved (it comprises acoustic, magnetic, and tactile stimuli). The SSR is dependent on integrity of peripheral sympathetic cholinergic pathways, as it is preserved in selective sympathetic adrenergic failure (as in dopamine $\beta$-hydroxylase deficiency), and is absent in pure (peripheral) autonomic failure (with sympathetic adrenergic and cholinergic failure) and in pure cholinergic dysautonomia. ${ }^{6}$

Although the SSR is an established technique for the study of peripheral sympathetic neuropathies, ${ }^{7-9}$ its clinical application in investigation of central sudomotor pathways is still debated. Although there is evidence in humans of arousal related cerebral centres that control the SSR, as reported in recent neuroimaging studies, ${ }^{10}$ previous reports ${ }^{11}{ }^{12}$ suggested that the SSR can be generated within the isolated spinal cord.

In addition to limitations related to the use of SSR in general, such as habituation and response variability, ${ }^{13}{ }^{14}$ the application of the technique in patients with spinal cord injury (SCI) needs to be considered in the light of two factors. The first is that with the wide range of neurological features of SCI, the segmental level and completeness of the lesion are of importance because of the thoracolumbar sympathetic outflow. Secondly, autonomic function is not taken into account in the International Standard for Neurological and Functional Classification of SCI. ${ }^{15}$

The aim of our study therefore was to assess the capability of an isolated spinal cord to generate SSR and determine the required levels of integrity of spinal cord and supraspinal connections. Electrical peripheral nerve stimulation only was used, as such a method offers the advantage of providing an objective measure of stimulus intensity and it can be compared in nerves above and below the neurological level of SCI.

We used electrically evoked SSR in healthy volunteers and patients with SCI clinically classified at different levels of lesion. Stimulation of peripheral nerves above and below the lesion was employed with simultaneous, bilateral recording from palmar and plantar sites.

\section{SUBJECTS AND METHODS}

\section{Subjects}

Twenty nine patients with SCI (21 male and eight female), mean age (SD) 37(16) years and 10 age matched healthy control subjects ( six male and four female), mean age (SD) 37(12) years participated in the study after giving informed consent. Ethical approval was obtained from the Aylesbury Vale local research ethics committee. None of the participants was taking any medication with known autonomic effects. The cause of SCI was traumatic in 22 patients, surgical in four, and infective in three The time from injury ranged from 3 to over 1000 weeks, with an average of 176 weeks from injury. Subjects with peripheral neuropathy, diabetes, carpal tunnel syndrome (evaluated with nerve conduction studies), traumatic brain injury (excluded by imaging and detailed neurological examination), or any neurological condition other than SCI were excluded from the study. Only subjects free from pain, pressure sores and clinical depression, and fit enough to be tested in a sitting position, were recruited. Subjects with clinical syndromes involving spinal cord hemisection (BrownSéquard) or peripheral nerve damage (cauda equina syndrome) were also excluded from the study. A detailed clinical

Abbreviations: SSR, sympathetic skin response; $\mathrm{SCl}$, spinal cord injury; ASIA, American Spinal Injury Association 
Table 1 Presence or absence of palmar and plantar SSR to stimuli above or below the lesion in patients with SCI

\begin{tabular}{|c|c|c|c|c|c|c|c|c|c|}
\hline \multirow[b]{2}{*}{ Patient No } & \multirow[b]{2}{*}{ Sex } & \multirow[b]{2}{*}{$\begin{array}{l}\text { SCI level and } \\
\text { ASIA grade }\end{array}$} & \multirow{2}{*}{$\begin{array}{l}\text { ASIA motor } \\
\text { score (max } \\
100)\end{array}$} & \multicolumn{2}{|c|}{$\begin{array}{l}\text { ASIA sensory score (max } \\
112 \text { ) }\end{array}$} & \multicolumn{2}{|l|}{ Palmar SSR } & \multicolumn{2}{|l|}{ Plantar SSR } \\
\hline & & & & Light touch & Pin prick & $\begin{array}{l}\text { Supra-lesion } \\
\text { stimuli }\end{array}$ & $\begin{array}{l}\text { Infra-lesion } \\
\text { stimuli }\end{array}$ & $\begin{array}{l}\text { Supra-lesion } \\
\text { stimuli }\end{array}$ & $\begin{array}{l}\text { Infra-lesion } \\
\text { stimuli }\end{array}$ \\
\hline 1 & M & C3 A & 10 & 14 & 12 & - & - & - & - \\
\hline 2 & M & C5 A & 14 & 20 & 17 & - & - & - & - \\
\hline 3 & $M$ & $\mathrm{C} 6 \mathrm{~A}$ & 7 & 26 & 27 & - & - & - & - \\
\hline 4 & M & $\mathrm{C} 6 \mathrm{~A}$ & 24 & 21 & 22 & - & - & - & - \\
\hline 5 & M & $\mathrm{C} 8 \mathrm{~A}$ & 40 & 38 & 36 & - & - & - & - \\
\hline 6 & $\mathrm{~F}$ & T2 A & 50 & 38 & 36 & - & - & - & - \\
\hline 7 & M & T2 A & 50 & 38 & 38 & ++ & - & - & - \\
\hline 8 & M & T4 A & 50 & 46 & 46 & - & - & - & - \\
\hline 9 & $M$ & T4 A & 50 & 46 & 46 & - & - & - & - \\
\hline 10 & M & T5 A & 50 & 48 & 48 & - & - & - & - \\
\hline 11 & $\mathrm{~F}$ & T5 A & 50 & 48 & 46 & - & - & - & - \\
\hline 12 & $M$ & T6 A & 50 & 54 & 54 & ++ & - & - & - \\
\hline 13 & $M$ & T6 A & 50 & 54 & 54 & - & - & - & - \\
\hline 14 & $\mathrm{~F}$ & T7 A & 50 & 58 & 58 & ++ & - & - & - \\
\hline 15 & $\mathrm{~F}$ & $\mathrm{~T} 11 \mathrm{~A}$ & 50 & 74 & 74 & ++ & - & - & - \\
\hline 16 & M & $\mathrm{T} 12 \mathrm{~A}$ & 50 & 82 & 83 & +++ & - & - & - \\
\hline 17 & M & LI A & 68 & 85 & 85 & +++ & - & + & - \\
\hline 18 & $M$ & C4 B & 36 & 33 & 16 & - & - & - & - \\
\hline 19 & $M$ & C4 B & 11 & 40 & 28 & - & - & - & - \\
\hline 20 & $\mathrm{~F}$ & C4 B & 14 & 44 & 18 & - & - & - & - \\
\hline 21 & M & C4 B & 11 & 27 & 28 & - & - & - & - \\
\hline 22 & M & $\mathrm{C} 4 \mathrm{C}$ & 48 & 104 & 104 & ++ & + & - & - \\
\hline 23 & M & C5 C & 78 & 100 & 97 & - & - & - & - \\
\hline 24 & M & C8 C & 74 & 68 & 66 & + & - & - & - \\
\hline 25 & $\mathrm{~F}$ & $\mathrm{TlC}$ & 29 & 28 & 28 & +++ & - & + & - \\
\hline 26 & $M$ & C4 D & 91 & 88 & 102 & +++ & +++ & +++ & +++ \\
\hline 27 & $M$ & C4 D & 90 & 94 & 94 & ++ & ++ & + & +++ \\
\hline 28 & $\mathrm{~F}$ & C6 D & 49 & 73 & 33 & ++ & + & +++ & - \\
\hline 29 & $\mathrm{~F}$ & TI D & 65 & 90 & 77 & ++ & - & - & - \\
\hline
\end{tabular}

neurological examination was performed on all subjects, after which they were divided into the following groups, based on the American Spinal Injury Association (ASIA) impairment scale $^{15}$ modified from Frankel et al ${ }^{16}$ :

\section{Group A}

Seventeen patients with SCI at various neurological levels from C3 to L1, with ASIA grade A (complete lesion: no motor function preserved below the lesion and no sensory function in the sacral segments S4-S5).

\section{Group B}

Four patients with SCI at neurological level Tl or above, with ASIA grade B (incomplete lesion, sensory preservation including the sacral segments S4-S5 but no motor preservation below the neurological level).

\section{Group C}

Four patients with SCI at neurological level Tl or above, with ASIA grade C. This includes incomplete lesion with some degree of motor preservation; more than half of key muscles below the neurological level have a muscle grade less than 3 on a scale of $0-5(0=$ no contraction; $5=$ normal strength $)$, according to Medical Research Council guidelines. ${ }^{17}$

\section{Group D}

Four patients with SCI at neurological level Tl or above, with ASIA grade D (incomplete lesion with some degree of motor preservation; more than half of key muscles below the neurological level have a muscle grade greater than or equal to 3 ).

\section{Group N}

Ten healthy control subjects.
The essential individual characteristics of all patients with SCI, including neurological level, ASIA motor and sensory scores, ${ }^{15}$ and time from onset of paralysis are shown in table 1.

\section{Investigations}

The SSR was recorded from palmar and plantar surfaces, both left and right, using neonatal ECG $\mathrm{Ag} / \mathrm{AgCl}$ surface electrodes (Arbo) with differential recording. One electrode was placed on the volar aspects of the hands or feet, on the skin above the third metacarpal or metatarsal bones (at $3 \mathrm{~cm}$ from the distal end). The second electrode was placed on the corresponding area of the dorsal aspect of the relevant hand or foot.

Optically isolated amplifiers were used with gain of 100 and filters at $-3 \mathrm{~dB}$ below 0.1 and above $100 \mathrm{~Hz}$, connected to a computer through an analog/digital interface. The signals were sampled at a rate of $200 \mathrm{~Hz}$. The traces were recorded from 0.5 seconds before to 8 seconds after the trigger stimulus. To minimise interference from spontaneous potentials, the operator monitored the baseline on an oscilloscope before releasing the electric stimulus, which was delivered at a varying time (30 to 35 seconds) after the previous stimulus.

During the test the subject sat in a chair or wheelchair and was relaxed but not asleep. The room temperature was maintained at $24 \pm 0.5^{\circ}$. The skin temperature of the upper and lower limbs was monitored and stayed constant from the beginning to the end of the test (intrasubject variability $<0.2{ }^{\circ} \mathrm{C}$ ). This protocol was followed as temperature is known to affect conduction velocity of unmyelinated sympathetic fibres. ${ }^{18}$

Each stimulus consisted of a single electric pulse (width 0.5 $\mathrm{ms}$ ). All subjects were stimulated at the median nerve at the wrist and peroneal nerve at the ankle. The stimulus intensity was set to 1.5 times the motor threshold of the stimulated nerve. In patients with complete SCI who did not have SSR to 


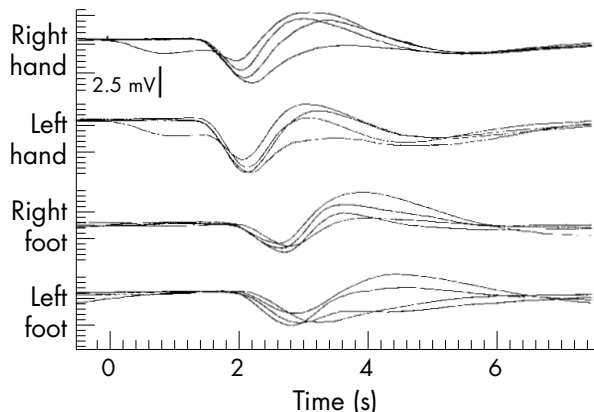

Figure 1 Superimposed SSRs to four consecutive right median nerve stimuli in a normal subject. Stimulus was applied at time 0 (artefact in right hand trace).

peroneal stimulation, the stimulation intensity was progressively increased up to levels equal to five times the motor threshold of normal subjects, unless the electric stimulus caused a muscle response diffuse enough to be noticed by the patient. The intensity of this stimulation would have been painful in healthy normal subjects. In tetraplegic patients, with a level of lesion higher than the root supply of the median nerve, the supraorbital nerve was also stimulated at the forehead, a technique used in patients with SCI to study the SSR with stimuli above the lesion level. ${ }^{19} 20$ A minimum of four events was recorded for each nerve and intensity level.

\section{Analysis}

The peak to peak amplitude and latency of SSR were measured. Only repeatable responses, consistent in latency, and temporal relation to the electric stimulus, were considered as electrically evoked SSRs. Potentials obtained in the presence of muscle spasms, passive or voluntary limb movement, deep inspiration, or coughing were excluded from the analysis. The latency (in ms) and amplitude (in $\mathrm{mV}$ ) of SSRs were measured for the first four trials to each nerve or, when the stimulus intensity was increased above 1.5 motor threshold, from the first trial producing a response. Values are expressed as mean (SD). Statistically significant levels reported compare a given group with controls, unless otherwise specified. A $\chi^{2}$ (or Fisher's exact test where appropriate) was used to compare the rate of responders/nonresponders (where responders refer to subjects presenting SSR), and analysis of variance (ANOVA) was used to compare latency and amplitude of responses in different groups.

\section{RESULTS}

The results reported refer to right palmar and plantar SSR to stimulation of the right median or supraorbital nerve above, or the peroneal nerve below the lesion level. Responses were also obtained from left nerve stimulation and left palmar/plantar recording. There was no significant difference between sides for response rate, amplitude, and latency.

\section{Control subjects}

All normal subjects had palmar and plantar SSR to median and peroneal nerve stimulation. For palmar SSR to median nerve stimulation, latency was 1476 (162) ms and amplitude 2.98 (1.5) mV. Plantar SSR to the same stimuli had longer latency (2231 (305) ms) and smaller amplitude (1.77 (1.11) $\mathrm{mV}$ ) than palmar SSR (fig 1).

For palmar SSR to peroneal nerve stimulation, latency was 1568 (SD 156) ms and amplitude 2.32 (SD 1.82) mV. Similarly to median nerve stimulation, plantar SSR to peroneal nerve stimulation had longer latency (2205 (215) ms) and smaller amplitude (2.29 (1.44) $\mathrm{mV})$ than palmar SSR to the same stimuli.

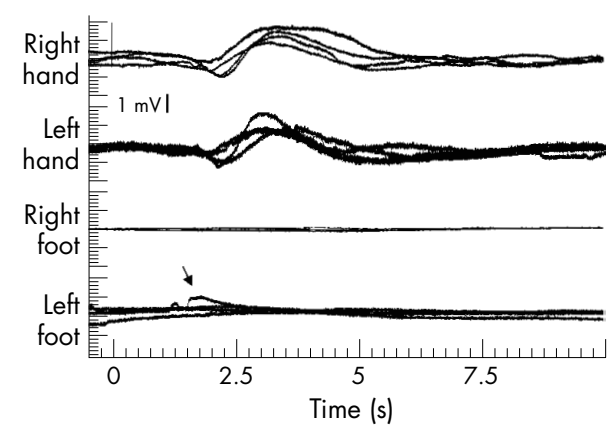

Figure 2 Superimposed SSRs to four consecutive right median nerve stimuli in a patient with $\mathrm{SCl}$ grade $\mathrm{A}$, level T2 (patient 7). There are repeatable, bilateral palmar responses of consistent latency whereas no plantar responses are recorded. Stimulus was applied at time 0 (artefact in right hand trace). An occasional artefact (arrow), inconsistent with the properties of an evoked response (non-repeatable, inappropriate latency to the stimulus) is present in the left foot trace.

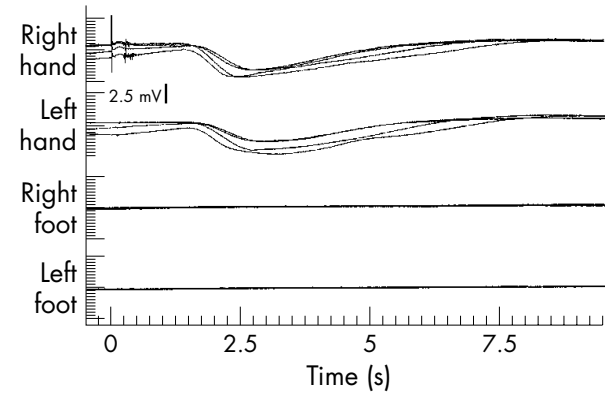

Figure 3 Superimposed SSRs to four consecutive right median nerve stimuli in a patient with complete, low thoracic SCI (patient 15 : level T 11, ASIA grade A). Stimulus was applied at time 0 (artefact in right hand trace). Repeatable palmar SSRs of normal amplitude are present in both sides. No plantar responses are present.

\section{Group A}

Palmar SSR to supralesion stimuli

In group A (complete SCI), the presence of a palmar SSR to supralesion stimuli (whether median or supraorbital nerve) was related to the level of the lesion (table 1 ). In only one of 11 patients with complete SCI above level T6 (patient 7, level T2) was a palmar SSR obtained (latency 1358 (124) ms; amplitude $2.18(0.25) \mathrm{mV}$; fig 2). One of the two patients with complete SCI at level T6 also had a response (patient 12: latency 1540 (140) ms; amplitude $1.67(0.86) \mathrm{mV})$. All patients with complete SCI below T6 had a palmar SSR (latency 1495 (91) ms; amplitude 4.42 (3.61); see fig 3). There were no significant differences in latency or amplitude between the SSR obtained in group A and in the control group ( $\mathrm{p}>0.5)$.

Plantar SSR to supralesion stimuli

A plantar SSR to supralesion stimuli (latency $2183 \mathrm{~ms}$, amplitude $1.1 \mathrm{mV}$; fig 4) was recorded in only one patient from group A (table 1, subject 17). That patient had SCI at level L1, the lowest in the group.

\section{Peroneal nerve stimuli}

No patients from group A had either palmar or plantar SSRs to peroneal nerve stimuli $(\mathrm{p}<0.001)$, even at five times normal motor threshold stimulation (fig 5).

\section{Group B}

No patients from group B (all with SCI at cervical level) had palmar or plantar SSRs to either median or peroneal nerve stimuli $(p<0.001)$, similarly to all patients with complete SCI with a comparable level of lesion. 


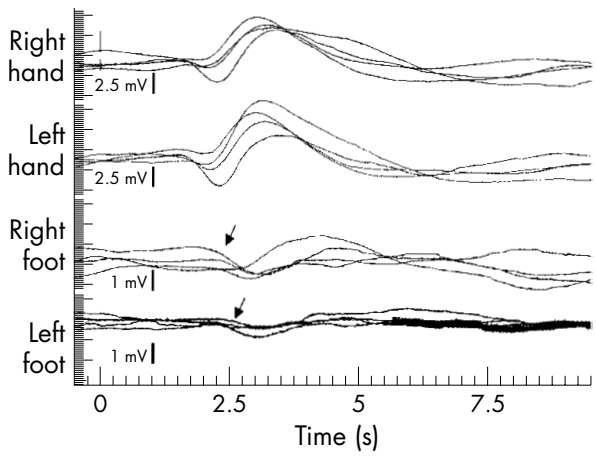

Figure 4 Superimposed SSRs to four consecutive right median nerve stimuli in a patient with SCl grade A, level L1 (patient 17). Stimulus was applied at time 0 (artefact in right hand trace). Bilateral SSRs of normal amplitude are evident in palmar sites. Repeatable, bilateral plantar SSRs of small amplitude are also evident (arrows); their latency, as expected for SSRs evoked at a more distant site, is slightly longer than palmar SSR.

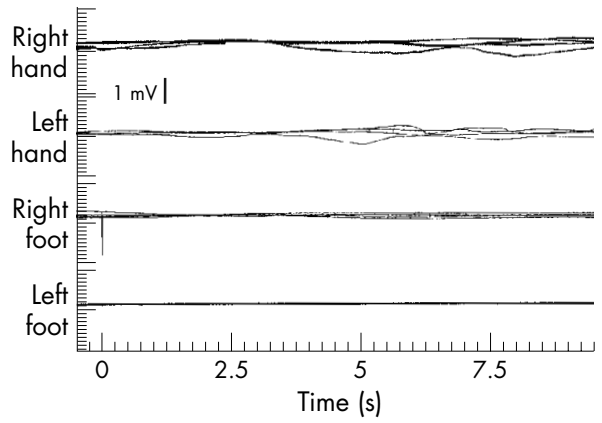

Figure 5 Superimposed SSRs to four consecutive right peroneal nerve stimuli in a patient with $\mathrm{SCl}$ at level $\mathrm{C} 3$, grade $\mathrm{A}$ (patient 1). Stimulus was applied at time 0 (artefact in right foot trace) with intensity levels equal to five times the motor threshold of normal subjects. No palmar or plantar responses are recorded.

\section{Group C}

In group C, palmar SSR to median nerve stimuli was recorded in three of four patients, with normal latency ( 1471 (60) ms; $\mathrm{p}>0.8$ ) and amplitude ( $1.95(0.82) \mathrm{mV} ; \mathrm{p}>0.4)$. One patient had plantar SSR to median nerve stimuli (patient 25: latency $2288 \mathrm{~ms}$; amplitude $0.25 \mathrm{mV}$ ), and one patient had palmar SSR to peroneal nerve stimuli (patient 22: latency $1598 \mathrm{~ms}$; amplitude $0.73 \mathrm{mV}$ ). No plantar SSRs to peroneal nerve stimuli were recorded in this group $(\mathrm{p}<0.001)$.

\section{Group D}

All patients from group D had palmar SSRs to median nerve stimuli (latency 1543 (212) ms, amplitude 2.33 (2.02) mV), and three of four patients had plantar SSRs to the same stimuli (latency 2330 (229) ms, amplitude 1.77 (1.11) mV). Palmar SSRs to peroneal nerve stimuli were recorded in three of four patients (latency 1661 (99) ms; amplitude 1.53 (0.63) $\mathrm{mV})$. Two of four patients had plantar SSRs to peroneal nerve stimuli (latency 2205 (215) ms; amplitude 2.55 (0.52)).

\section{DISCUSSION}

In our study of complete SCI at different levels there was no evidence of isolated spinal cord sudomotor responses to electrical stimuli. In none of our patients with complete SCI (ASIA grade A) was a palmar or plantar SSR obtained in response to electrical stimuli applied below the lesion, regardless of lesion level or stimulation intensity. An SSR to stimuli below the lesion was also absent in patients with SCI of grade B (in whom the sensory stimulus was conducted to the brain stem and sensory cortex, as they could feel the activating stimuli).
Conversely, some patients with a degree of preservation of both motor and sensory function (ASIA grades C and D) had palmar and plantar SSRs to stimuli below the lesion. The mean amplitude of these responses was smaller than normal, although the difference was not statistically significant. The latency of the SSRs was within the normal range, suggesting that the responses were conducted through the same neurological pathways used in normal subjects.

A plantar sudomotor response to lower extremity noxious stimuli has been reported in patients with functionally complete cord transection at cervical and thoracic levels. ${ }^{11}{ }^{12}$ Such responses, however, were not recorded consistently, and the duration of electrical stimulation (pulse trains of $0.5 \mathrm{~s}$ ) did not favour a precise analysis of the latency. Also, in those two studies, patients with SCI were only broadly classified by a single neurological level, and the partial preservation of sensory and motor pathways in segments below the lesion level was not documented using ASIA groups or quantified using ASIA motor and sensory scores. Therefore, the possibility of some degree of supraspinal connection and partial preservation of pathways below the lesion was not excluded in those patients.

Our results in healthy control subjects demonstrated no significant differences between median and peroneal nerve stimulation in SSR amplitude or response rate. In patients with SCI, however, differences in response rate and SSR amplitude could be seen according to the degree of preservation of the stimulated sensory pathway. In general, supra lesion stimuli produced closer to normal responses than did peroneal nerve stimuli. A similar effect was shown by groups with a different degree of motor preservation (group D had closer to normal responses than group C). Habituation of the SSR, which has been suggested to have a non-neurological, peripheral component at sweat gland level, ${ }^{21} 22$ might contribute to these differences. Patients with SCI often have trophic skin disturbances including severe skin dehydration, especially in the lower limbs. However, we did not find a significant reduction in amplitude over the first four responses either in patients with SCI or in normal subjects, indicating that habituation did not affect our results. We suggest therefore that a quantitative effect of damage to both sensory and motor pathways is responsible for the differences in SSR amplitude between the different ASIA groups.

Overall, our findings indicate that connections with supraspinal structures are essential for evoking SSRs. The association of preserved somatomotor function and presence of SSRs might be explained by the anatomical proximity, in the spinal cord, of the sympathetic preganglionic nuclei (intermediolateral columns from level T1 to L2) to the anterior horn nuclei.

Our data show that the SSR, which is an indicator of sympathetic cholinergic function, is very often abolished or impaired (both in palmar and plantar sites) in SCI above level T6 (depending on the completeness of SCI and ASIA level). On the other hand the sympathetic adrenergic function at these levels of lesion is released from supraspinal control and is typically hyperreactive to stimuli coming from below the lesion (skeletal muscle spasms, bladder or rectal activity, labour, noxious stimuli such as anal fissures). The condition leads to autonomic dysreflexia, to which patients with SCI above T6 (especially complete) typically are known to be prone. $^{23}$ Dissociation between autonomic dysreflexia and presence of palmar SSR was also reported in previous studies by Curt et al..$^{24}$ This might reflect differences in the sympathetic neural control of the cardiovascular and the sudomotor system.

Our data from patients with complete SCI indicate that supraspinal connections to the upper thoracic cord segments are required for the palmar SSR, possibly with borderline levels around T6. This does not mirror the levels required for the somatic innervation of the upper limbs via dorsal and ventral 
roots, and is more probably related to distribution of preganglionic sympathetic neurons to the sympathetic ganglionic chain. These findings are consistent with the findings of Yokota $e t a^{26}$ that the SSR probably reflects the integrity of central preganglionic pathways as well as peripheral postganglionic activity.

It is possible that supraspinal connections down to Ll might be essential for plantar SSR, but we had too few patients with relevant injuries to be certain. The high prevalence of nerve root damage (cauda equina syndromes) in lumbar SCI limits the availability of patients with purely central lesions at lumbar level.

An apparently exceptional finding was seen in patient 7, who had a palmar SSR to supralesion stimuli despite having an ASIA grade A lesion at level T2. These responses, which had smaller amplitude than normal, were bilateral, repeatable, clearly related to the stimulus, and consistent in latency and waveform shape with the SSR recorded in normal volunteers. The neurological examination indicated a sensory level at T2 bilaterally, involving all types of sensation, with partial preservation at level T3. No motor or sensory function was detected below this level. The patient had no documented episodes of autonomic dysreflexia. A provocative test was performed using suprapubic bladder stimulation and blood pressure recording, ${ }^{23}$ which was negative. The injury was in its chronic phase (67 weeks) and therefore, if a predisposition to develop autonomic dysreflexia were present, it would have been manifested by the time of the study. This might be consistent with the possibility that the lesion was not complete with regard to the sympathetic pathways. It must be emphasised that ASIA classification does not take into account autonomic pathways, and partially preserved connection of some segments below the highest damaged level is not unusual, even in SCI classified as complete. It is possible that our subject had partially preserved sympathetic pathways for a few segments below the lesion, sufficient to produce palmar SSR of decreased amplitude. However we cannot exclude the possibility that, in a minority of cases, partial palmar responses might be present even when the sympathetic pathways are totally interrupted at a higher level than T6.

In conclusion, our study has defined the inability of the isolated spinal cord to generate SSR. It emphasises the potential of electrically evoked SSR in investigation of the neural control of sweating and central sudomotor pathways in SCI, particularly at a thoracic level. Furthermore, it might provide a useful technique in assessing the completeness of lesion in SCI and improve the current classification methods for neurological level and completeness of SCI, which are based on the somatic nervous system only.

\section{ACKNOWLEDGEMENTS}

This work was supported by the International Spinal Research Trust. PC is the recipient of a Nathalie Rose-Barr PhD Scholarship.

\section{Authors' affiliations}

P Cariga, M Catley, P H Ellaway, Department of Sensorimotor Systems, Division of Neuroscience and Psychological Medicine, Imperial College School of Medicine at Charing Cross Hospital, Fulham Palace Road, London W6 8RF, UK

P Cariga, C J Mathias, Neurovascular Medicine Unit, Imperial College School of Medicine at St. Mary's, Praed Street, London W2 INY, UK

G Savic, H L Frankel, National Spinal Injuries Centre, Stoke Mandeville Hospital, Mandeville Road, Aylesbury HP2 1 8AL, UK

\section{REFERENCES}

1 Shahani BT, Halperin JJ, Boulu $P$, et al. Sympathetic skin response: a method of assessing unmyelinated axon dysfunction in peripheral neuropathies. J Neurol Neurosurg Psychiatry 1984;47:536-42.

2 Knezevic W, Bajada S. Peripheral autonomic surface potential. A quantitative technique for recording sympathetic conduction in man. J Neurol Sci 1985:67:239-51.

3 Uncini A, Pullman SL, Lovelace RE, et al. The sympathetic skin response: normal values, elucidation of afferent components and application limits. J Neurol Sci 1988;87:299-306.

4 Rossini PM, Opsomer RJ, Boccasena P. Sudomotor skin responses following nerve and brain stimulation. Electroencephalogr Clin Neurophysiol 1993;89:442-6.

5 Linden D, Weng Y, Glocker FX, et al. Sympathetic skin responses evoked by magnetic stimulation of the neck: normative data. Muscle Nerve 1996;19:1487-9.

6 Magnifico F, Misra VP, Murray NM, et al. The sympathetic skin response in peripheral autonomic failure: evaluation in pure failure, pure cholinergic dysautonomia and dopamine- $\beta$-hydroxylase deficiency. Clin Auton Res 1998;8:133-8.

7 Soliven B, Maselli R, Jaspan J, et al. Sympathetic skin response in diabetic neuropathy. Muscle Nerve 1987;10:711-6.

8 Solders G, Andersson T, Persson A. Central conduction and autonomic nervous function in HMS'N I. Muscle Nerve 1991; 14:1074-9.

9 Sagliocco L, Sartucci F, Giampietro O, et al. Amplitude loss of electrically and magnetically evoked sympathetic skin responses in early stages of type 1 (insulin-dependent) diabetes mellitus without signs of dysautonomia. Clin Auton Res 1999;9:5-10.

10 Critchley HD, Elliott R, Mathias CJ, et al. Neural activity relating to generation and representation of galvanic skin conductance responses: a functional magnetic resonance imaging study. J Neurosci 2000;15:3033-40

11 Fuhrer MJ. Analysis of electrodermal evidence for a para-medullary afferent tract in patients with a transection of the thoracic spinal cord. $J$ Neurol Neurosurg Psychiatry 1971;34:281-8.

12 Fuhrer MJ. Effects of stimulus site on the pattern of skin conductance responses evoked from spinal man. J Neurol Neurosurg Psychiatry 1975;38:749-55

13 Hoeldtke RD, Davis KM, Hshieh PB, et al. Autonomic surface potential analysis: assessment of reproducibility and sensitivity. Muscle Nerve 1992;15:926-31.

14 Aramaki S, Kira Y, Hirasawa Y. A study of the normal values and habituation phenomenon of sympathetic skin response. Am J Phys Med Rehabil 1997;76:2-7

15 Maynard FM Jr, Bracken MB, Creasey G, et al. International standards for neurological and functional classification of spinal cord injury. American Spinal Injury Association. Spinal Cord 1997;35:266-74.

16 Frankel HL, Hancock DO, Hyslop G, et al. The value of postural reduction in the initial management of closed injuries of the spine with paraplegia and tetraplegia. Paraplegia 1969;7:179-92.

17 Medical Research Council. Aids to the examination of the peripheral nervous system. Medical research council memorandum 45. London: Her Majesty's Stationary Office, 1976.

18 Deltombe T, Hanson P, Jamart J, et al. The influence of skin temperature on latency and amplitude of the sympathetic skin response in normal subjects. Muscle Nerve 1998;21:34-9.

19 Nair KP, Taly AB, Arunodaya GR, et al. Sympathetic skin response in myelopathies. Clin Auton Res 1998;8:207-11

20 Obach V, Valls-Sole J, Vila N, et al. Sympathetic skin response in patients with lateral medullary syndrome. J Neurol Sci 1998: 18; 15555-9

21 Cariga P, Catley M, Ellaway PH. Habituation of the sympathetic skin response in normal human subjects: the effect of repeated electrical stimulation of peripheral nerves. J Physiol 2000;523:221.

22 Cariga P, Catley M, Mathias CJ, et al. Adaptation of sympathetic skin responses in man: effects of repeated electrical stimulation of peripheral nerves on duration, latency, and amplitude [abstract]. Clin Auton Res 2000;10:160.

23 Mathias CJ, Frankel HL. Autonomic disturbances in spinal cord lesions. In: Mathias CJ, Bannister R, eds. Autonomic failure. A textbook of clinical disorders of the autonomic nervous system. Oxford: Oxford University Press, 1999;494-513

24 Curt A, Weinhardt C, Dietz V. Significance of sympathetic skin response in the assessment of autonomic failure in patients with spinal cord injury. J Auton Nerv Syst 1996;6:175-80.

25 Curt A, Nitsche B, Rodic B, et al. Assessment of autonomic dysreflexia in patients with spinal cord injury. J Neurol Neurosurg Psychiatry 1997;62:473-7.

26 Yokota T, Matsunaga T, Okiyama R, et al. Sympathetic skin response in patients with multiple sclerosis compared with patients with spinal cord transection and normal controls. Brain 1991;114:1381-9. 